\title{
Kernos
}

Revue internationale et pluridisciplinaire de religion grecque antique

$16 \mid 2003$

Varia

\section{S. ALCock et al. (éds), Pausanias. Travel and Memory in Roman Greece}

\section{Yves Lafond}

\section{OpenEdition \\ Journals}

Édition électronique

URL : http://journals.openedition.org/kernos/853

DOI : $10.4000 /$ kernos.853

ISSN : 2034-7871

\section{Éditeur}

Centre international d'étude de la religion grecque antique

\section{Édition imprimée}

Date de publication : 1 janvier 2003

Pagination : 380-384

ISSN : 0776-3824

\section{Référence électronique}

Yves Lafond, «S. ALcock et al. (éds), Pausanias. Travel and Memory in Roman Greece », Kernos [En ligne], 16 | 2003, mis en ligne le 14 avril 2011, consulté le 24 septembre 2020. URL : http:// journals.openedition.org/kernos/853; DOI : https://doi.org/10.4000/kernos.853 
scolaire, dont un des buts, comme le dit l'A. elle-même, est de présenter le texte et de regrouper les informations qui y sont contenues, il reste encore bien des zones d'ombre autour de ces textes qui « offrent un vaste champ d'études ».

Carine Van Liefferinge (Université libre de Bruxelles

Olli Salomies (éd.), The Greek East in the Roman Context. Proceedings of a. colloquium organised by the Finnish Institute at Athens, May 21 and 22, 1999, Helsinki, 2001. 1 vol. $17,5 \times 25 \mathrm{~cm}$, II +217 p., 5 pl. (Papers and Monographs of the Finnish Institute at Athens, 7). ISBN : 951-98806-0-7.

L'analyse de l'impact du pouvoir romain sur la Méditerranée orientale - en ce compris la Grèce - a connu ces dernières années une recrudescence d'intérêt et donc une efflorescence de publications, que ce soient des monographies, des articles ou des actes de colloques. Ces Actes sont un bon reflet de l'actualité d'une telle préoccupation et réunissent un bel ensemble de spécialistes, confirmés ou à venir. Les questions religieuses ne sont toutefois pas nombreuses, ce qui justifie la brièveté de cette notice. Épinglons donc la contribution de J.-L. Ferrary (Rome et la géographie de l'bellénisme: réflexions sur. 'bellènes' et 'panbellènes' dans les inscriptions d'époque romaine) qui touche notamment à la question du Panhellénion; les deux inscriptions qui constituent le départ de cette étude ont été découvertes dans le sanctuaire de Claros et touchent à des actions

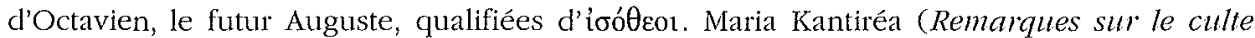
de la domus Augusta en Achaïe de la mont d'Auguste à Néron) offre une brève contribution à l'étude du culte dynastique dans la province d'Achaïe, en tentant une datation des diverses avancées en cette matière. Mika Kajava (Vesta and Atbens) pose quant à lui un très intéressant problème d'histoire religieuse : la Vesta romaine, que l'interpretatio graeca identifie comme une Hestia, avait-elle une place à Athènes, et, si oui, quel était la part de l'idéologie proprement romaine - au-delà d'un simple opportunisme politique des Athéniens - qui avait présidé à cette implantation? La démonstration est longue et parfois touffue, mais l'hypothèse d'une localisation sur l'Acropole, dans le monoptère d'Auguste et de Rome à partir de 12 av. J.-C. a de bons arguments en sa faveur. S. Follet et D. Peppas Delmousou (Les dédicaces cborégiques d'époque flavienne et antonine à Athènes), dans une contribution essentiellement épigraphique, fournissent le corpus (avec quelques photographies excellentes) remis à jour de ces inscriptions qui étaient autant de dédicaces en prose ou en vers illustrant les monuments supportant le trépied donné au vainqueur des choeurs dithyrambiques des Grandes Dionysies. P. Themelis (Roman Messene. The Gymnasium) rend compte de ses fouilles sur ce site et fait notamment référence à un certain nombre de statues divines qui s'élevaient dans le gymnase et à des inscriptions honorifiques mentionnant des fondations religieuses ou des sacrifices. D'autres contributions concernent le rapport de la 'Grèce de l'est' à la mémoire de la république romaine (C.P. Jones), les élites municipales des colonies romaines d'Achaïe (A.D. Rizakis), la famille de Théophane de Mytilène (K. Buraselis), la première province romaine de Syrie (M. Sartre, avec en annexe la liste des gouverneurs de Syrie entre 64 et 31), les inscriptions honorifiques pour les sénateurs romains en 'Grèce de l'est' (O. Salomies) et les cognomina latins dans cette région (H. Solin). Des index soigneux complètent utilement cet ensemble.

Vinciane Pirenne-Delforge (FNRS - Université de Liège)

Susan Alcock, John F. Cherry, Ja's Elsner (éds), Pausanias. Travel and Memory in Roman Greece, Oxford, University Press, 2001.1 vol. $16 \times 24 \mathrm{~cm}$, xII +379 p., 27 fig. ISBN : 0-19-512816-8.

Comme le soulignent les éditeurs dans leur préface, le présent volume trouve sa légitimation dans le regain d'intérêt dont bénéficie depuis peu Pausanias, un auteur long- 
temps cité et utilisé par fragments ${ }^{1}$, en particulier comme base à des études archéologiques $^{2}$, ou comme point d'appui à une histoire religieuse de la Grèce archaïque et classique, alors que la lecture de la Périégèse fait découvrir une ouvre ancrée dans une époque - la Grèce des Antonins - et où sont perceptibles des principes de méthode qui donnent à chacun des dix livres conservés et à l'ensemble une cohérence plus grande que certains voudraient le croire. Deux colloques récents ${ }^{3}$ ont d'ailleurs envisagé sous des angles divers cette question des modalités d'une lecture de Pausanias, et témoignent tout particulièrement de ce qu'on peut appeler un processus de réhabilitation auquel on ne peut manquer d'associer l'ouvrage de Christian Habicht ${ }^{4}$ ainsi que les éditions italienne et française de l'œuvre'.

Le sous-titre de l'ouvrage semble prendre en compte clairement en tout cas l'ancrage de l'œurve de Pausanias dans la Grèce de son temps et place au cour de la réflexion des thématiques - celles du voyage et de la mémoire - assurément propres à renouveler notre approche du texte du Périégète.

Des travaux récents avaient déjà permis d'en juger : ceux de S. Alcock ${ }^{6}$ elle-même qui, en cherchant à analyser les effets de la conquête et de la provincialisation, étudie tout particulièrement les paysages et leurs transformations et, à travers un réexamen des données tant archéologiques que littéraires, s'efforce de retrouver les traces d'un dialogue Rome / Grèce; ceux aussi de J. Elsner dont les articles successifs consacrés au problème de l'identité chez Pausanias ${ }^{7}$ ont contribué à montrer que, loin d'être un simple guide touristique, le texte de la Périégèse pouvait servir à comprendre comment les Grecs ont cherché à se définir par rapport à un passé dont le poicls est important lorsqu'il s'agit d'identité culturelle ${ }^{8}$, par rapport aussi à la politique contemporaine dans une Grèce

1 Il est significatif, par exemple, que la traduction et le commentaire fournis par E. Meyer en 1954 (réédités en 1967) n'aient comporté que les parties descriptives de la Périégèse.

2 Il faut mentionner à ce sujet les thèses de G. Daux, Pausanias à Delphes, Paris, 1936; G. Roux, Pausanias en Corintbie, Paris, 1958; M. Josr, Pausanias en Mégalopolitide, Bordeaux, 1972 et les commentaires de N.D. Papachatzis, parus en 1965 et qui étaient alors en cours de réédition.

3 J. Bingen (dir.), Palisanias bistorien, Vandœuvres/Genève, 1996 (Entretiens de la Fondation Haldt, 41); D. KNoepfler, M. PiÉrart (éds), Éditer; traduire, commenter Pausanias en l'an 2000 (Actes du colloque de Neuchâtel et de Fribourg, 18-22 septembre 1998), Genève, Droz, 2001.

¿ Paru pour la première fois en allemand, en 1985, sous le titre Pausanias und seine Bescbreibung Griechenlands et réédité récemment en anglais : Pausanias' Guide to Ancient Greece, Berkeley/Los Angeles/Londres, 1998.

5 En cours depuis 1982 (Fondazione Lorenzo Valla : livres I à VII parus) et 1992 (Collection des Universités de France : livres I, V à VIII parus).

6 Voir "Archaeology and Imperialism: Roman Expansion and the Greek City", Journ. of Mediter: Arcbaeology 2 (1989), p. 87-135; Graecia Capta. The Landscapes of Roman Greece, Cambridge, 1993; "Greece : a Landscape of Resistance?", in D.J. MatTingly (éd.), Dialogues in Roman Imperialism. Power, Discourse, and Discrepant Experience in the Roman Empire, Portsmouth/Rhode Island, 1997 (JRA, Suppl. 23), p. 103-115: cet article reprend le concept de «paysage» mais veut montrer que, parmi les facteurs susceptibles de rendre compte des transformations et de l'évolution d'une «province », il ne faut pás négliger de prendre en considération les conditions géographiques locales et les attentes du pouvoir impérial (avec le problème des interactions entre la politique impériale et les pratiques locales), tout comme la place historique de l'Achaïe comme patrie des Grecs.

J. Elsner, "Pausanias : a Greek Pilgrim in the Roman World", Past and Present 135 (1992), p. 3-29; "From the Pyramids to Pausanias and Piglet : Monuments, Travel and Writing ", in S. Goldhill, R. Osborne (éds.), Ant and Text in Ancient Greek Culture, Cambridge, 1994, p. 224-254; Ant and the Roman Viewer; Cambridge, 1995, chap. 4: "Viewing and Identity: the Travels of Pausanias; or, a Greek Pilgrim in the Roman World", p. 125-155. Dans ce dernier ouvrage, reprenant la même démarche que dans l'article de 1992, l'A. part du principe que, les monuments et les ceuvres d'art étant des incarnations d'une histoire affimant la présence du passé, étudier la façon dont ils sont perçus par une collectivité ou par un individu permet d'envisager la question des identités dont ils constituent une expression et d'étudier, en particulier, dans le cas de Pausanias, le conflit entre des aspirations générales communes à la culture gréco-romaine et des revendications individuelles.

8 D. Musti ("La struttura del discorso storico in Pausania", dans Entretiens Pausanias, p. 21, 23 et 29) considère que la Périégèse joue un rôle significatif dans la construction d'une identité 
devenue province romaine et où, pour la société grecque, la nécessité d'entretenir la mémoire pouvait apparaître comme un moyen de sauvegarder son identité ${ }^{1}$.

La composition globale du livre présenté ici fait apparaître trois grandes sections, organisées selon des rubriques générales. La première est consacrée à l'auteur et à son œuvre : J. Elsner y souligne à juste titre la nécessité de considérer le texte de Pausanias comme une construction littéraire répondant à un projet d'ensemble cohérent, et appuie sa démonstration sur l'exemple de la description d'Olympie aux livres V et VI ${ }^{2}$; E. Bowie, au fil d'une mise au point sur divers aspects de la chronologie et des sources d'inspiration de la Périégèse, aborde la difficile question de la réception de Pausanias dès l'Antiquité. Le genre périégétique et le thème du voyage sont plus spécifiquement traités dans deux contributions consacrées l'une (C.P. Jones) aux guides de Pausanias, c'est-à-dire à cette catégorie de personnages chargés d'informer dans les cités les voyageurs de passage, l'autre (I. Rutherford) à la notion de pèlerinage, ce qui permet d'étudier les liens entre le «tourisme » et le sacré et d'en montrer la spécificité dans le contexte de la Seconde sophistique.

Les quatre études que regroupe, sous un titre très général («Studies and Comparisons »), la deuxième section, la plus développée, proposent une approche de la Périégèse visant à dégager les principes de méthode de son auteur et à souligner l'influence qu'exerce sur le texte le contexte social et culturel : l'exemple particulier de la description du coffre de Cypsélos au livre $\mathrm{V}$ et l'évocation du passé messénien au livre IV servent ainsi de références pour une analyse de la méthode et des stratégies interprétatives de Pausanias dans le domaine de la description d'œuvres d'art (A.M. Snodgrass) et de la narration historique ( $\mathrm{S}$. Alcock), tandis que A. Cohen et I. Porter prennent en compte respectivement, dans la lecture plus globale qu'ils font de la Périégèse, les courants artistiques et littéraires susceptibles d'avoir servi de toile de fond à la composition de l'œuvre, aussi bien dans sa tonalité que dans le choix de certains thèmes. On notera l'intérêt et la richesse de ces deux dernières contributions, l'une explorant les liens de la Périégèse avec les représentations de l'espace et plus particulièrement du voyage dans l'art d'époque hellénistique et romaine, l'autre mettant l'accent sur la notion de sublime appliquée au texte de Pausanias et judicieusement mise en rapport avec les thèmes de l'identité et de la mémoire.

La troisième et dernière section ( "Nachleben», mais la signification donnée à ce terme n'apparaît pas clairement et l'on se demande si «Rezeption » n'aurait pas été plus approprié) affronte le problème de la réception de Pausanias en envisageant plus particulièrement l'utilisation et l'interprétation que firent de la périégèse quelques lecteurs célèbres du $\mathrm{xIX}^{\mathrm{e}}$ siècle : le colonel Leake (J.M. Wagstaff), Lewis R. Farnell (J. Henderson) et Jane Harrison (M. Beard), à une époque marquée par une floraison exceptionnelle d'ouvrages (éditions, traductions, commentaires, études diverses) suscités par le texte de Pausanias - au point que M. Beard (p. 229) peut évoquer « the nineteenth-century Pausanias boom ». La lecture de Pausanias, on le voit à travers ces essais, contribua en tout cas à orienter le développement des études classiques, en favorisant le croisement d'approches relevant de multiples domaines - l'histoire, l'étude des religions et des mythes, mais aussi l'anthropologie et l'archéologie.

On saura gré d'ailleurs aux éditeurs du présent volume d'avoir mis en évidence l'intérêt d'une lecture pluridisciplinaire du texte de Pausanias, puisque les points de vue rassemblés ici émanent aussi bien d'historiens que de littéraires, d'historiens de l'art, d'archéologues et d'anthropologues. Cela n'est pas sans donner une saveur particulière à l'ensemble, qui se distingue aussi par le parti pris de faire suivre chaque section de «commentaires »: on regrettera peut-être que ces brefs essais n'aient pas été mis plus

culturelle grecque définie comme « une sorte de réponse créative au défi lancé par l'affirmation de la domination romaine ». Les concepts d'identité, d'autonomie et de liberté constitueraient la base idéologique du discours historique de Pausanias.

1 Qu'il me soit permis de signaler en outre à ce sujet ma thèse d'Habilitation, soutenue en novembre 2002 : La mémoire des cités dans le Péloponnèse d'époque romaine (II' siècle av. J.-C.-III" siècle ap.J.-C.).

2 On pourra ajouter l'étude d'A. Jacquemin, "Pausanias, témoin de la religion grecque dans le sanctuaire d'Olympie", in A PAsquier (éd.), Olympia, Paris, 2001, p. 181-213. 
systématiquement en rapport avec la matière présentée dans chaque grande section et n'aient pas servi finalement à instaurer un dialogue plus constructif qui aurait abouti à une mise au point plus nette sur quelques grands thèmes. La plupart de ces chapitres constituent plutôt des sortes de pauses dans la réflexion et, sous forme d'analyses globales ou rattachées à des thématiques particulières, font écho aux sujets abordés dans le reste de l'ouvrage : les contributions de M. Torelli, D. Konstan, P. Cartledge et J.F. Cherry portent ainsi sur les critères axiologiques, les goûts et les intentions de Pausanias, et soulignent leurs implications sur notre lecture de la Périégèse. Trois contributions cependant se rattachent directement au contenu de la section qu'elles viennent clore : outre celle de S. Bann sur une allusion de Chateaubriand à Pausanias et celle de J. Elsner, sous forme de « coda », dans la troisième section, sur l'importance des références à Pausanias pour le peintre Joseph Michael Gandy au début du $\mathrm{xIx}^{e}$ siècle, il faut signaler la contribution suggestive de B. Bergmann sur les rapports entre la «Grèce imaginaire » de Pausanias et la représentation des paysages dans l'art et la littérature de l'Italie d'époque impériale, qui apporte un prolongement utile au contenu déjà riche de la deuxième section.

Dans l'ensemble, tout se passe comme si les auteurs de ce volume avaient eux-mêmes recherché cette «bigarurre » si chère déjà aux écrivains de la Seconde sophistique. Or, il me semble que plusieurs des contributions de ce volume auraient gagné à être resserrées davantage sur les problématiques annoncées par le titre et notamment autour du thème de la mémoire qui, avec le problème de l'oubli qui lui est étroitement lié, notamment lorsqu'il y a manipulation de la mémoire, principalement par voie «idéologique »', occupe une place privilégiée dans le texte de Pausanias.

L'étude de C.P. Jones sur "Pausanias et ses guides" vise certes à mieux cerner l'identité des exégètes que consulte Pausanias en quête d'informations sur les traditions locales des cités qu'il parcourt, mais ne souligne pas assez, me semble-t-il, le rôle que jouent ces personnages comme dépositaires de la mémoire civique. La contribution de S. Alcock consacrée au problème du passé messénien permet quant à elle de souligner l'exemplarité du livre IV de la Périégèse pour une réflexion sur le caractère livresque et sélectif de la mémoire que Pausanias transmet à travers sa présentation du passé des cités. Mais s'agissant par exemple d'Aristoménès, élevé au rang de héros par les Messéniens, il est dommage que l'importance du personnage ne soit pas mise en rapport avec les pratiques contemporaines des Messéniens, attestées à la fois par le texte même de la Périégèse et la documentation épigraphique, Alcock préférant traiter de l'influence de la lecture de Pausanias sur l'enquête archéologique en Messénie pour expliquer le déséquilibre qui s'est instauré, dans ce domaine, entre l'attention portée aux périodes extrêmes de l'histoire messénienne (époques mycénienne et romaine) et celle accordée aux périodes archaïque et classique, beaucoup plus limitée. L'importance qu'occupe dans la mémoire civique Aristoménès, personnage issu du lointain passé de la cité, s'accorde pourtant avec l'image qu'en propose Pausanias, soucieux, comme une étude non citée par Alcock l'a justement montré ${ }^{2}$, d'associer aux chefs messéniens dont il reconstitue l'histoire des valeurs caractéristiques d'un icléal moral qui est celui des dirigeants d'époque romaine.

Dans son bref essai au titre pourtant prometteur ( Sparta's Pausanias : Another Laconian Past »), P. Cartledge préfère revenir de son côté sur la question de la valeur de Pausanias comme témoignage sur l'histoire de la Grèce archaïque et classique plutôt que d'envisager le problème de ce qui, dans les Laconica, relève de l'idéologie civique contemporaine. Je ne suis pas sûr pourtant qu'on puisse affirmer sans plus ample enquête, comme le fait Cartledge (p. 170) « qu'en matière de religion spartiate antique - archaïque et classique, aussi bien que contemporaine de son époque - le témoignage de Pausanias est simplement indispensable ". La mise en perspective du témoignage de Pausanias par rapport au genre périégétique et par rapport aux courants sophistiques, réalisée ici sous forme de trois contributions stimulantes, aurait gagné aussi à faire plus nettement ressortir

1 Et l'on voit chez un auteur comme Pausanias à quel point les stratégies de l'oubli ou ce qu'on peut appeler une idéologisation de la mémoire sont rendues possibles par le travail de configuration narrative (d'où les études de C. Calame) à l'ouvre dans la Périégèse comme il l'était dans les oraisons funèbres de l'Athènes classique étudiées par N. Loraux.

2 J. Auberger, "Pausanias et le livre IV : une leçon pour l'Empire", Pboenix 54 (2000), p. 255281. 
les implications de ces discours sur la définition des valeurs helléniques et, partant, la spécificité de Pausanias.

Chaque contribution est accompagnée de notes (regroupées en fin de volume) qui exploitent une bibliographie bien fournie (il faut remplacer cependant Aupert P. par Piérart M. comme éditeur de l'ouvrage Éditer, traduire, commenter Pausanias en l'an 2000, paru chez Droz en 2001). Des figures en noir et blanc, un index des passages cités de Pausanias et un index général complètent utilement le tout. Mais la carte de Grèce qui ouvre le volume, sans échelle ni aucune indication de relief, n'est guère suggestive.

Yves Lafond (Université d'Artois)

H.F.J. Horstmanshoff, H.W. Singor, F.T. Van Straten, J.H.M. Strubbe (éds), Kykeon. Studies in Honour of H.S. Versnel, Leiden, Brill, 2002. 1 vol. $16 \times 24,4 \mathrm{~cm}, 228$ p. (Studies in Greek and Roman Religion, 142). ISBN : 90-04-11983-3.

Henk Versnel est une des figures marquantes de l'histoire des religions classiques de notre époque. Devenu émérite en septembre 2000, après avoir enseigné à l'Université de Leiden depuis 1978, il a été honoré par ses collègues par le biais d'un Colloque qui s'est tenu à Leiden en mai 2000, dont les Actes sont intitulés Kykeôn, une « potion magique » en hommage à un des meilleurs connaisseurs du monde de la magie, des rites, des sacrifices, tant sur le versant grec que sur le versant romain ${ }^{1}$. On regrettera du reste que le volume, au demeurant riche et intéressant, ne contienne pas une liste de ses publications.

W. Burkert propose, en Introduction, une mise au point passionnante sur «'Mythos und Ritual' im Wechselwind der Moderne » (p. 1-22). Il met en perspective les recherches sur les rapports entre mythe et rite depuis le début du $\mathrm{xx}^{\mathrm{e}}$ siècle (y compris son fondamental Homo necans paru en 1972) jusqu'à l'époque post-moderne.

A. Chaniotis, en partant des Daidala de Béotie, étudie les « Ritual Dynamics ». Connues par le biais de Plutarque et Pausanias, ces festivités impliquaient directement les rapports entre Héra et Zeus. Le problème est donc de savoir si l'on a affaire à un bieros gamos ou non. En confrontant les diverses analyses, qui insistent tantôt sur le mythe, tantôt sur le rite, l'A. montre bien que la synthèse est à portée de la main, sans forcer la documentation (Putting the pieces togetber), en tenant surtout compte de la stratification chronologique, de «stades » (les Allemands parlent d'Entwicklungsgeschichte) dans le développement du complexe mythico-rituel de Platées. Une telle lecture est susceptible de s'appliquer à bien d'autres fêtes religieuses grecques.

L'essai de B. Gladigow affronte la question de la pluralité culturelle et religieuse de l'Europe, hier comme aujourd'hui, et notamment la manière de concevoir les rapports entre monothéismes et polythéismes, ainsi que l'apport de la koinè religieuse méditerranéenne et de la religion impériale romaine à l'histoire de la religion européenne qui doit être étudiée comme un ensemble, et non pas de manière sectorielle.

R. Gordon, dans la ligne des intérêts scientifiques de Versnel, propose une étude sur les textes magiques gréco-égyptiens. Il y est question du pouvoir magique des noms, de la création de nouvelles techniques, de la manipulation des textes, des images, autant d'éléments destinés à accroître la puissance de l'acte magique. On signalera aux lecteurs une étude récente sur le même sujet, du point de vue socio-linguistique: P. Poccetti, "Manipolazione della realtà e manipolazione della lingua: alcuni aspetti dei testi magici dell'Antichità", in R. Morresi (a cura di), Linguaggio - Linguaggi. Invenzione - Scoperta, Rome, 2002, p. 11-59.

F. Graf répond brillamment à la question «What is new about Greek Sacrifice? », une mise au point synthétique fort intéressante qui tend à confirmer les thèses de Meuli et Burkert sur le sacrifice comme mise à mort de l'animal.

H.J. de Jonge centre son attention sur « The Apocalypse of John and the Imperial Cult », en se rattachant à une étude de Versnel parue en 1988, en rapport avec une publication de F. Millar. La question était de savoir dans quelle mesure le refus de la part

1 Le sommaire de ce livre est présenté infra, p. 392. 